\title{
Enjeux, frontières et limites de l'autofiction italienne
}

\author{
Marco Mongelli \\ Università à Degli Studi di Siena
}

Malgré la sortie de Fils en 1977, l'Italie des années 1980 ignorait encore le mot «autofiction» et la pratique qu'il désignait. Voici qu'un quart de siècle plus tard, tout le monde en parle, souvent pour le disqualifier.

Que s'est-il passé pendant ce temps? De méconnue à motvalise, l'autofiction a vécu et vit, en Italie, une singulière aventure, qu'il vaut peut-être la peine de retracer et de regarder de plus près. Ce qui saute aux yeux du chercheur (ou même du simple lecteur attentif) est que personne en Italie n'a jamais considéré l'autofiction comme une pratique digne d'être étudiée de façon spécifique et approfondie, comme on le fait 
pour n'importe quelle nouveauté littéraire. Que ce soit pour son ambiguïté structurelle ou pour l'évidente contradiction dans les termes que l'appellation exprimait, l'autofiction s'est vue, en France et ailleurs, abordée à la fois comme pratique à la mode et comme rien de vraiment nouveau, comme quelque chose qui existait déjà depuis longtemps dans la littérature et dont il n'y avait pas lieu de s'enthousiasmer. Mais alors qu'en France, les poéticiens et les théoriciens de la littérature ainsi que les écrivains s'interrogeaient sérieusement sur le défi doubrovskien, en Italie, on a commencé à parler d'autofiction sans se préoccuper de ce qui s'écrivait ou se théorisait à ce sujet audelà des Alpes. À l'exception de Martemucci (2008), tous les travaux, même les plus récents, n'abordent la question que de façon transversale et comparative, sans effectuer un examen attentif du phénomène italien.

Pourtant, les témoignages éclatants de la vivacité et de la productivité du dispositif autofictionnel dans la littérature italienne contemporaine ne sont pas rares. Bon nombre de romans déjà canonisés chez nous peuvent être inscrits dans le domaine de l'autofiction. Partons donc des textes spécifiques, de ces fictions ou non-fictions à l'air étrange pour mettre en relief les caractéristiques de fond de l'usage autofictionnel italien, son évolution dans les années 1990 jusqu'à la maturité acquise ces toutes dernières années, où trois textes, à mon avis exemplaires, ont donné à l'autofiction italienne une visibilité et un élan décisif.

Dans cette optique, il est sans doute nécessaire de clarifier les termes de la question pour ne pas risquer de réutiliser l'autofiction comme passe-partout ni d'aplatir toute narration du je au champ autofictionnel. En somme, qu'est-ce 
que l'autofiction? Un genre (ou un sous-genre) qui forme le texte entièrement ou bien un registre qui peut être utilisé de façon locale et stratégique? Il s'agirait plutôt, à mi-chemin, d'un dispositif, à savoir d'un ensemble de figures et de procédés littéraires cohérents qui visent à un résultat esthétique déterminé. Néanmoins, ce dispositif, bien que "bâtard», possède depuis longtemps des contraintes strictes, des conditions nécessaires quoique non suffisantes: la triple identité onomastique entre auteur-narrateur-protagoniste et la formulation d'un pacte ambigu avec le lecteur, pacte ni totalement romanesque ni totalement autobiographique. Pour parler d'autofiction, il faut donc, au moins, être en présence d'un récit autodiégétique où l'auteur demande au lecteur à la fois de le croire et de se méfier. Un récit, somme toute, où l'indécidabilité entre ce qui est fictif et ce que ne l'est pas est inévitable, mais où pourtant toute «information» est intratextuelle ou paratextuelle.

\section{L'autofiction italienne : le début}

S'il est possible de parler d'autofiction au sens large pour la littérature italienne déjà à partir des années 1980, il est toutefois nécessaire d'affirmer que l'importance acquise par les narrations du soi il y a trente ans et qui s'est poursuivie dans les années 1990, n'a qu'un lien apparent avec l'expérience de l'autofiction, qui en effet souvent la transcende.

Il faut donc tout d'abord distinguer un certain biographisme générationnel sans structure chez Tondelli (Altri libertini, 1980), Culicchia (Tutti giù per terra, 1994) ou Brizzi 
(Jack Frusciante è uscito dal gruppo, 1994), d'une vraie attitude autofictionnelle, où le matériel biographique est manipulé de manière explicite et ne se contente pas de produire une simple identification de la part du lecteur, mais agit pour «multiplier les niveaux » de lecture.

Pour reconstruire rétrospectivement le chemin du genre et ainsi retrouver des caractéristiques communes, il faut donc relire de façon différente toute une série d'écritures qui peuvent, aujourd'hui seulement, se rattacher à l'autofiction. Il s'agit de textes où les auteurs mettent en scène un matériel narratif objectivement autobiographique tout en modifiant (ou cachant) leur nom ou en le gardant tel quel. Pour marquer une évolution de la sensibilité du dispositif, on peut distinguer les textes aux identités non parfaites, c'est-à-dire où le nom du protagoniste ne coïncide pas explicitement avec celui de l'auteur (et du narrateur), de ceux où la triple identité est au contraire bien évidente et bien marquée. De cette façon nous arriverons aux années zéro du XXI ${ }^{\text {e }}$ siècle, où nous assistons à un mélange de formes, de styles et de registres qui accomplit une véritable nouvelle construction littéraire : l'autofiction au sens strict. Parus dans l'espace de quelques années, Troppi paradisi (2006) de Walter Siti, Italia De Profundis (2008) de Giuseppe Genna et Spaesamento (2010) de Giorgio Vasta, traduit et publié en 2012 chez Gallimard sous le titre Dépaysement, sont trois romans qui peuvent être lus et comparés surtout à travers le dispositif autofictionnel.

Ce bref aperçu que je vais entreprendre nous permettra de voir comment, à partir d'une autobiographie plus ou moins conventionnelle, les écrivains vont complexifier la relation biographique qu'ils instaurent entre eux-mêmes et l'instance 
énonciatrice. Si l'autofiction est aussi le résultat de l'évolution de l'autobiographisme dans l'époque postmoderne, le passage n'en est pas moins graduel.

Si Aldo Busi, dans son premier roman, Seminario sulla gioventù (1984), sorte de biographie romancée, présente comme protagoniste un double de lui-même, dans Casanova di se stessi (2000), il complique le mécanisme identitaire en nommant son protagoniste Aldo Subi et passant donc du «double» à une «moitié complémentaire ». Avec Kamikaze d'Occidente (2003) de Tiziano Scarpa, le roman autobiographique prend la forme du journal intime. Le but de Kamikaze est de rassembler deux instances narratives totalement différentes qui se structurent autour de la forme du journal intime romancé, le configurant ainsi comme l'une des modalités possibles de l'autofiction. Pour La cultura enciclopedica dell'autodidatta (2006), Davide Bregola utilise un « simple » pseudonyme, une sorte d'alter ego. De cette manière, sans claire identité nominale, on est donc en présence d'une autobiographie à la manière de Svevo-Zeno. Cependant, cette stratégie permet à l'auteur de raconter tout ce qui arrive hors de la scène, en faisant du «je» une image de la foule. Ce procédé d'universalisation du « je » est bien commun et permet à l'autofiction de réussir là où une autobiographie plus classique aurait échoué. Une autre relation possible est celle que met en scène Emanuele Trevi dans Senza verso. Un'estate a Roma (2005), où le narrateur-protagoniste n'est jamais nommé mais peut être identifié à l'auteur par l'usage de plusieurs « effets de vie».

On trouve un autre topos répandu de cette écriture protoautofictionnelle dans la forme du journal intime «fiction- 
nalisé ». Dans Maggio Selvaggio (2001), par exemple, Edoardo Albinati l'utilise pour rapporter une expérience d'enseignement en prison. Mais si la fiction, l'invention, ne concernent pas la biographique mais le simple changement de noms, de dates, de détails, à quel titre peut-on parler d'autofiction? Nous savons bien que la fictionnalisation n'est pas forcément liée au contenu, mais aussi à la mise en intrigue. Dans Maggio selvaggio, en effet, l'acte de fictionnalisation relève du montage qui organise le matériel narratif, véritablement autobiographique, de façon à produire un récit «fictif». Il est évident qu'aujourd'hui, aucun écrivain ne peut compter sur un récit linéaire de sa propre vie. L'autobiographie ne peut jamais être sincère, car l'acte de parler de soi ne distingue pas le vrai du faux. Voilà donc comment le journal intime devient un outil efficace, permettant de sonder le réel, lorsqu'il est opportunément manipulé de manière fictive.

Le parcours suivi par Mauro Covavich témoigne bien de cette évolution des formes. Si, dans A perdifiato (2003) et dans Fiona (2005), où évoluent des doubles et alter ego, les protagonistes et leurs histoires semblent seulement faire allusion à l'écrivain et à sa vie, dans Prima di sparire (2008), l'auteur accomplit une opération pleinement autofictionnelle, faisant de son protagoniste l'écrivain "Mauro Covacich ». Cependant, cela ne suffit pas, parce que l'exhibition au ton humble de l'écrivain est dépourvue de la violence typique des meilleures autofictions. Pour être réellement efficace, une autofiction doit instaurer une contradiction fructueuse entre le «je » qui écrit et sa représentation romanesque, la contradiction propre de notre contemporain médiatisé et soumis à la fiction. 
Voilà donc le thème dominant de la production autofictionnelle italienne, décliné en différents motifs, et toutefois omniprésent, incisif et quelque peu dangereux.

Dans Il bambino che sognava la fine del mondo (2009) d'Antonio Scurati, le motif d'intérêt demeure dans son pastiche de faits divers, d'articles publiés sur le web et dans les journaux, et, évidemment, de fiction et d'autobiographie. Le thème central est en effet une violente critique du monde de l'information et des médias qui créent de faux scoops pour alimenter la peur et s'en nourrir après. Le frénétique enchaînement de réalité et fiction, de chronique et d'autobiographie, sert à décrire la genèse des hystéries collectives et l'effacement d'un regard critique, désamorcé par l'envahissant système médiatique.

Il libro della gioia perpetua (2010) d'Emanuele Trevi, présente la triple identité auteur-narrateur-protagoniste et représente une radicalisation du motif autobiographique. Le roman est un hybride entre journal intime et invention narrative parce que le personnage «Emanuele Trevi » mène la narration en étant protagoniste absolu, tandis que le réel est manipulé de manière fictionnelle. Si le cadre "réaliste» confirme une tendance très répandue dans l'autofiction italienne, le caractère "psychanalytique » est par contre très propre à Trevi. En effet, le texte fonctionne comme une autoanalyse, à la fois interprétation et réinvention du soi, bien loin donc du point de départ, une trentaine d'années avant.

Il faut mentionner aussi le cas particulier de Gomorra, de Roberto Saviano, le livre "culte» sur le phénomène de la Camorra. Il représente un très bon exemple de la confusion et de l'insuffisante conscience des nouvelles formes littéraires 
présentes en Italie. En effet, les critiques n'ont pas trouvé initialement, pour un texte aussi original dans notre langue, une « étiquette » plus convenable que « autofiction ». Plutôt literary non-fiction qu'autofiction, ce pseudo-reportage ne peut être inscrit dans notre domaine parce que l'auteur ne met pas en discussion son "je » et son vécu et ne se réinvente pas dans l'écriture. Au contraire, son regard est ferme et son identité bien forte. D'ailleurs, le pacte est absolument antifictionnel et il n'y a aucune superposition de fictif et de vrai, aucune ambiguïté ni contradiction.

\section{Maturité et nouveaux enjeux}

Pourtant, un texte qu'on pourrait qualifier de «vraie» autofiction était sorti en 1994 et s'intitulait Scuola di nudo. Récemment traduit en français et publié aux Éditions Verdier sous le titre Leçons de nu, il s'agit du premier roman de Walter Siti, professeur à l'École normale supérieure de Pise et critique littéraire. La stratégie narrative particulière, unie à un discours explicite sur la contemporanéité, fait de ce texte une sorte de manifeste symbolique de l'autofiction italienne. Le personnage «Walter Siti » fonctionne comme « un je expérimental » parce que son rôle est justement celui d'expérimenter et de raconter les « virus » de notre époque, la prolifération d'obsessions et de pathologies dont on ne voit que les épiphénomènes et qu'on voudrait, au contraire, saisir politiquement. Je dis que c'est une sorte de manifeste parce que les autofictions qui vont suivre seront telles à partir d'une constatation évidente sur le changement socio-anthropologique de notre pays. Il y a 
autofiction dans la mesure où il $\mathrm{y}$ a la perception d'un irréversible envahissement du public (en tant que produit des médias, et donc aussi comme ensemble de spectateurs) dans la vie privée, qui se fait extrême et exemplaire, manifestation douloureuse de la dérive du présent. L'écriture autofictionnelle trouve son véritable enjeu et lance son défi à partir de la constatation que l'autobiographie et le roman à la première personne sont désormais inutilisables. Il faut un écrivain qui s'engage avec son propre nom et son propre corps et qui décrit de l'intérieur une déformation qu'il ne serait pas capable de nommer autrement.

Selon Siti, on peut distinguer deux types d'autobiographie : une comme but, l'autre comme moyen. La première est l'autobiographie classique, celle qui illustre la vie de celui qui parle et en rapporte les faits avec la conviction qu'ils sont exemplaires ou instructifs; la seconde est un artifice typiquement romanesque, utilisé par qui écrit comme prétexte pour regarder le monde. C'est précisément par l'intermédiaire de ce dispositif que le « je » peut se narrer en fonction des gens, du peuple (italien, occidental ou autre) et devenir allégorie du présent. Voici donc la fonction cognitive de l'autofiction: si l'autobiographie classique a pour but d'exalter l'auteur, l'autobiographie comme moyen a pour but de l'exploiter jusqu'à la consomption. "Autobiographie comme moyen» pourrait donc bien être une des définitions de l'autofiction.

Il y a par conséquent une autre condition de possibilité d'existence de l'autofiction: non seulement l'identité onomastique - nécessaire, mais non suffisante - mais aussi les dispositifs de «figures cohérentes» qui certifient et encouragent la réception purement autofictionnelle du texte. 
Les autofictions italiennes sont donc caractérisées par la mise en discussion du vécu, passé et présent, de l'auteur-narrateurprotagoniste. Les plus récentes accomplissent un acte de démystification $\mathrm{du}$ voile que le bavardage médiatique superpose à notre réalité, désormais impossible à distinguer du « récit permanent » des médias.

Il est important de remarquer qu'à partir de la célèbre définition de Doubrovsky, l'autofiction reste en France très liée à un refus du faux et du mensonge ; par contre, en Italie, seule la réinvention d'une identité sur base fictive peut étendre la notion d'autofiction à plusieurs niveaux de lecture: littéraire, socio-anthropologique, historique-philosophique, ou encore psychanalytique.

De cette vision découle une définition brillante de l'autofiction que donne Siti : "autobiographie de faits jamais arrivés» (1999, je traduis). En renversant l'axiome de Doubrovsky de «fiction d'événements et de faits strictement réels ", Siti montre que la structure romanesque, en prenant le dessus sur la mémoire, remplit l'autobiographie de faits inventés et jamais vécus. L'autofiction devient alors un roman à part entière, qui, chez les meilleurs écrivains, se charge de toutes ses valeurs positives. Le regard du « je » qui vit, narre et écrit est attentif et toutefois fallacieux. L'autofiction est donc une vérification de soi, une sorte d'ordalie sans appel, non plus un moment d'auto-célébration. La sincérité ne passe plus par la plainte mais par l'invention : fictionnaliser une identité est un acte de sincérité où un « je » réel est traduit par l'autofiction en un « je » irréel.

Toutefois, pour comprendre pleinement les opérations artistiques de Siti, de Genna et de Vasta, il faut relever 
l'intention " politique » de ces écritures. Le récit assumé par ce «je expérimental » devient immédiatement un acte politique, voire d'analyse et de critique de notre contemporanéité, non par les moyens de l'essai ou du pamphlet, mais par ceux de la fiction. Les marques de cette "époque berlusconienne» que l'on vit depuis trente ans et qui dépassent la simple période de présence politique sont désormais profondes, causes et conséquences, symboles et cauchemars.

Siti, à travers le monde des fictions télévisées et du sousmonde des banlieusards prostitués homosexuels de la Rome d'aujourd'hui (l'envers de celle de Pasolini), Genna via l'asphyxiant succédané de réalité que sont les villages de vacances d'été, Vasta moyennant les types humains les plus représentatifs de la ville de Palerme, visent précisément à faire une fresque de cette Italie. Dépersonnalisation et transpersonnalisation $\mathrm{du}$ « je » sont les instruments permettant de rendre un récit particulier significatif d'un pays et d'une époque. Comme d'habitude, les meilleurs documents d'un présent sont les documents esthétiques, voire fictionnels.

\section{Limites et nouvelles potentialités}

Il est néanmoins juste d'observer que, par rapport aux nombreux défis que l'autofiction se propose, il y a autant de risques d'échecs. Dans la relation dynamique instaurée avec la fiction télévisée et l'infotainment dominant, la majorité des œuvres ne réussit pas à renverser ce paradigme dominant. La mimesis, même parodique, des attitudes, du langage et des aspirations des personnages télévisés, célèbres ou non, est très 
dangereuse. Faute de talent ou de regard original sur le monde, on finit par mimer une pratique sans s'en distancier. On est dans l'exhibition guidée, et donc conformiste, des affects, des émotions, du vécu. Il ne suffit pas de se mettre à nu, de se culpabiliser, de se dévaloriser, de s'autonommer digne symbole de temps indignes. Il faut inventer, narrer: il faut donc de la fiction. Diverses œuvres parabiographiques parues en Italie dans les quinze dernières années témoignent paradoxalement d'une moindre capacité de représentation littéraire des expériences vécues. À une volonté de subjectivation du regard ne correspond pas une profondeur cognitive universelle, entraînant ainsi le risque de renverser la direction de «l'auto » vers soi-même. Si la réalité n'est au service d'aucune structure, elle devient fait divers insignifiant.

Telle est donc la plus grande limite de l'autofiction de nos jours : ne pas inscrire le récit d'une vie dans un projet fort de description du réel. Au contraire, la limite que soulignent toujours ses détracteurs, le manque de clarté théorique qui empêcherait une définition stricte et originale, peut se transformer en avantage si l'on considère que l'autofiction peut se construire sur n'importe quel dispositif narratif.

En effet, même en considérant l'autofiction dans son sens le plus large, c'est-à-dire comme mise en fiction de la vie personnelle de l'auteur, on ne peut pas ne pas reconnaître la valeur de l'innovation. La pratique intrinsèquement « métissante » de l'autofiction brise le champ littéraire et nous oblige à reconsidérer les notions de "genre littéraire » et de «fiction-non-fiction». Le dispositif autofictionnel peut donc s'insérer dans toute narration et on peut le repérer dans tout texte qui en présente les marques particulières. De plus, ce qui 
fait d'un roman parabiographique une «vraie » autofiction est l'engagement politique explicite de son auteur, qui peut se servir de la forme essai comme du journal intime, du reportage comme du portrait.

Comme le dit Giglioli, on peut relever, au sein de la production narrative italienne récente, une tentative consciente et souvent théorisée de «forcer le genre à être quelque chose de différent de ce qu'il est» (2011, p. 28; je traduis). Les normes formelles ou structurelles qui dirigent la composition d'un texte de genre, qu'il s'agisse d'un roman noir, historique ou de science-fiction, sont soumises ces dernières années à une intention allégorique-politique. De même, l'autofiction modifie les pratiques consolidées de l'écriture (ainsi que les réceptions) de l'autobiographie et du roman à la première personne, les hybride avec l'essai, le journal intime, le reportage, et met en place, dans les cas les plus réussis, un discours complexe et puissant.

En effet, si le genre peut être pensé en tant que pur dans l'abstraction théorique, c'est toujours de façon hybride qu'il agit dans les textes : son action est soumise à différentes possibilités de déformations, car elle peut subir des procédés de combinaison, de mutations fonctionnelles et d'adaptations à d'autres codes expressifs. " En plus si on considère l'intention d'un texte comme la tension active entre virtualité et réalisation, le genre littéraire peut bien se définir comme le signe de cette intention. » (Conte, 1986, p. 10 ; je traduis) On peut considérer le genre comme un moyen de signification inscrit dans le texte, qui donne forme et sens au discours mais aussi des instructions à celui qui lit. Le genre sectionne le 
champ littéraire, choisit un type de discours et de langage et communique au lecteur le sens de son opération.

L'importance du lecteur dans le procès de déploiement des diverses significations d'un texte est donc désormais établie. Il pourrait donc être question de réception et non de «substance» autofictionnelle. Loin de vouloir échapper au débat, souvent il semble qu'une recherche sur une ontologie textuelle de l'autofiction ne fait qu'aboutir à un cercle vicieux. Sans arrêter les investigations, on peut à juste titre affirmer que, dès que les termes et la pratique autofictionnelles sont entrés dans notre littérature, il s'est créé un cadre de réception qui favorise la lecture d'un texte parabiographique en tant qu'autofiction. Elle devient donc de nouveau un dispositif, un outil de questionnement des textes, non le seul, mais dans certains cas le plus efficace.

De même, il est possible d'être poussé à lire comme autofictionnelle toute œuvre qui reflète en quelque sorte son auteur. On peut donc assister à une sorte de quête dans la littérature du passé. Mais si on admet que les choses n'existent qu'une fois nommées, alors pas d'autofiction avant sa formulation: l'intentionnalité d'un texte n'est pas négligeable. Toutefois, certains romans du passé peuvent être lus à partir du dispositif autofictionnel, qui, de cette façon, réussit à éclairer des aspects jusque-là méconnus.

Pour démontrer la fécondité d'une reconsidération critique-interprétative des textes à partir de leurs intentions de genre, Nicola Lagioia (2011) a réfléchi sur La Pelle de Malaparte, relevant dans le roman des pratiques textuelles et des postures de l'auteur semblables à celles que nous définissons comme autofictionnelles. 
D'ailleurs, la littérature n'est pas le seul domaine artistique où ce dispositif s'est énoncé: depuis quelques années, on commence à parler d'autofiction visuelle. En considérant l'autofiction surtout en tant que "journal intime», on peut essayer de rédiger une liste de films qui peuvent être classifiés comme autofictions. Les tout premiers qu'on pourrait nommer sont Caro Diario (1993) - paru en France justement sous le titre de Journal intime - et Aprile (1998) de Nanni Moretti. Ils témoignent du fait qu'en Italie aussi, cette modalité de se fictionnaliser et de se narrer a produit des résultats très importants.

En conclusion, on peut revenir sur la question du concept d'autofiction, sur l'opportunité qu'il nous donne d'analyser le réel sous divers points de vue. Symptôme central de notre Zeitgeist, de cette époque sans centre et sans direction, l'autofiction est devenue la clé privilégiée pour lire une contemporanéité narcissique et exhibitionniste, fictive au point de passer pour réelle. Mais dans le but de faire fonctionner cette notion, il serait nécessaire de repartir des romans, de les relire et de ne pas cesser de les interroger. Il y a souvent là-dedans une perturbation profonde des dichotomies traditionnelles de notre pensée: seule l'autofiction mine les associations naturelles de fiction comme mensonge et de réalité comme vérité. La fiction n'étant pas une feinte, elle peut avoir une remarquable portée de vérité. 


\section{Bibliographie}

ConTE, Gian Biagio. (1986), «L'amore senza elegia : i Remedia amoris e la logica di un genere », dans Ovidio, Rimedi contro l'amore, a cura di Lazzarini Caterini, Venezia, Marsilio

DARRIEUSSECQ, Marie. (1996), «L'autofiction, un genre pas sérieux », Poétique, n 107, p. 369-380

DE MONTREMY, Jean-Maurice. (2002), «L'aventure de l'autofiction », Le Magazine Littéraire, n 409, p. 62-63

FOREST, Philippe. (2001), Le Roman, le je, Paris, Pleins Feux

GASPARINI, Philippe. (2004), Est-il Je? Roman autobiographique e autofiction, Paris, Seuil

GenetTe, Gérard. (1991), Fiction et diction, Paris, Seuil

GENNA, Giuseppe. (2008), Italia de profundis, Roma, minimum fax

Giglioli, Daniele. (2001), Senza Trauma, Macerata, Quodlibet.

LAGIOIA, Nicola, (2011) Il libro maledetto, minima\&moralia, 11 gennaio, <http://www.minimaetmoralia.it/?p=3610>.

LAOUYEN, Mounir. (2001), L'Autofiction: une réception problématique, Fabula, <http://www.fabula.org/forum/colloque99/208.php>

Lejeune, Philippe. (1975), Le Pacte autobiographique, Paris, Seuil 
LUCAMANTE, Stefania. (1998), «Le scelte dell'autofiction: il romanzo della memoria contro il potere della storia », Studi Novecenteschi, $\mathrm{n}^{\circ}$ 56, p. 367-381.

MARTEMUCCI, Valentina. (2008), «L'autofiction nella narrativa italiana degli ultimi anni. Una rassegna critica e un incontro con gli autori », Contemporarea, ${ }^{\circ}$ 6, p. 159-188.

MonGELLI, Marco. (2011), « Mentire raccontandosi : l'autofiction nel romanzo italiano degli ultimi anni», : $<$ http://www.academia.edu/1578017/Mentire raccontando si lautofiction nel romanzo italiano degli ultimi anni>

REGNIER, Thomas. (2002), « De l'autobiographie à l'autofiction : une généalogie paradoxale », Le Magazine Littéraire, $\mathrm{n}^{\circ}$ 409, mai, p. 64-65

SiTI, Walter. (1999), « Il romanzo come autobiografia di fatti non accaduti », Italies - Narrativa, p. 109-115

-. (2006), Troppi paradisi, Torino, Einaudi

VASTA, Giorgio. (2010), Spaesamento, Roma-Bari, Laterza

\section{Résumé}

Cet article aborde le sujet de l'autofiction en Italie en essayant, à travers l'analyse des formes littéraires particulières que cette tradition a produites, d'enquêter sur la spécificité qu'elle a acquise au cours des trente dernières années. Il s'agira donc de vérifier les enjeux, les frontières et les limites de cette pratique narrative en tant que forme d'écriture nouvelle par rapport au contexte spécifique italien. Si les écrivains qui se sont risqués dans une forme de narration si hasardeuse sont hétérogènes et 
parfois opposés quant à leur âge, leur style, leur ambition, il est possible de vérifier les raisons d'un choix qui, de façon plus ou moins consciente, s'avère obligé. En comparant les textes dans lesquels est mis en place un dispositif autofictionnel, nous dégagerons une intention commune aux auteurs : représenter la société italienne contemporaine et ses mutations anthropologiques. Il semble donc qu'à un certain moment, ce nouveau dispositif importé de France de manière vague et approximative était plus efficace que les formes classiques de narration pour saisir l'Italie d'aujourd'hui. Toutefois, à partir de quand pouvons-nous parler d'autofiction en Italie? Y-a-t-il des précédents significatifs dans la production romanesque du XX siècle? De plus, les libertés que les écrivains prennent sont-elles toujours nécessaires? En passant en revue les textes protoautofictionnels de la tradition romanesque italienne des trente dernières années, nous essayerons de montrer les formes et les thèmes propres au contexte national ainsi que leurs potentialités et leurs limites. L'ambiguïté intrinsèque de la voix qui énonce le récit révèle une potentialité inédite pour l'écriture italienne, mais aussi un risque redoutable. Il faut être conscient que se raconter à travers l'écriture narrative signifie non seulement modifier les faits mais aussi réinventer la perception qu'on a de soi-même et du monde que l'on habite. Ainsi, le mensonge narratif, c'est-à-dire la fiction, peut-il dire la vérité mieux que n'importe quel récit factuel.

\begin{abstract}
This article discusses the topic of autofiction in Italy trying, through the analysis of particular literary forms this tradition has produced, to examine the specificity that it has acquired over the past thirty years. The purpose is to verify issues, boundaries and limits of this narrative practice as a new form of
\end{abstract}


writing in relation to the specific Italian context. The writers who have experimented such a hazardous narrative form are heterogeneous and sometimes opposite in terms of age, style and ambition; it is therefore possible to question the reasons for a choice that, more or less consciously, proves to be compulsory. By comparing texts in which an autofictional device is employed, we will point out an intention shared by the authors: representing contemporary Italian society and its anthropological mutations. It seems that at a certain point this new device, imported from France in a vague and approximate manner, became more effective than conventional narrative forms to understand today's Italy. Nonetheless, since when can we talk of autofiction in Italy? Are there some significant precursory cases in the novelistic production of the twentieth century? Moreover, are the liberties taken by writers always needed? In reviewing the proto-autofictional texts of the Italian novelistic tradition of the past thirty years, we will try to show forms and themes specific to the national context, as well as their potential and their limits. The inherent ambiguity of the voice narrating the story reveals unprecedented potential for Italian writing, but also a dangerous risk. In fact, the author who talks about himself through narrative writing has to be aware that this means not only to change the facts, but also to reinvent the perception of himself and of the world he inhabits. Thus, the narrative lie, that is to say fiction, can tell the truth better than any factual narrative. 